\title{
Fluencia a alta temperatura de policristales con tamaño de grano nanométrico de YTZP dopados con diferentes cantidades de fase vítrea
}

\author{
C. LORENZO-MARTíN, D. GÓMEZ-GARCÍA, A. MUÑOZ-BERNABÉ, A. DOMÍNGUEZ-RODRÍGUEZ \\ Departamento de Física de la Materia Condensada, Universidad de Sevilla. Sevilla. España
}

\begin{abstract}
Muestras de YTZP (policristales de óxido de circonio dopados con itria) con tamaños de grano nanométrico y diferentes cantidades de fase vítrea han sido sometidas a experiencias de fluencia en un rango de temperatura entre $1150^{\circ} \mathrm{C}$ y $1200^{\circ} \mathrm{C}$, con objeto de estudiar el comportamiento mecánico de dichos materiales. Para conocer los mecanismos de deformación puestos en juego se ha realizado el estudio microestructural de las muestras deformadas y no deformadas mediante Microscopía Electrónica de Transmisión (MET). Se ha demostrado que la presencia de fase vítrea da lugar a un significativo descenso de la resistencia a la fluencia. No obstante, los parámetros característicos de la deformación ( $\mathrm{n}$ y Q) no se ven alterados, obteniéndose como mecanismo de deformación el deslizamiento de las fronteras de grano.
\end{abstract}

Palabras Claves: nanocerámicos, YTZP, fase vitrea.

\section{High temperature creep of nanometric YTZP polycristals with different glassy phase contents}

Nano-sized YTZP (Yttria Tetragonal Zirconia Polycrystals) samples with different amounts of a glassy phase have been crept in a range of temperature between $1150^{\circ} \mathrm{C}-1200^{\circ} \mathrm{C}$. A macroscopic characterization of the mechanical behaviour as well as the microstructural analysis by Transmission electron Microscopy (TEM) of both the as-received and deformed samples have been carried out. The glassy phase accounts for a significant softening while the deformation parameters values $(n, Q)$ are not altered with respect to those measured in non-doped specimens of the same material with the same average grain size.Grain boundary sliding has been proved to be the deformation mechanism.

Keywords: YTZP, glassy phase, nanoceramics.

\section{INTRODUCCIÓN}

Los materiales cerámicos nanométricos han despertado un gran interés en las últimas décadas debido, principalmente, a sus potenciales aplicaciones, inducidas por su facilidad para ser moldeados en formas complejas, así como por sus inesperadas propiedades.

Los materiales cerámicos nanométricos de circonia tetragonal dopada con itria (YTZP), ha sido muy estudiados en el rango submicrométrico gracias a la superplasticidad exhibida (1). De la escasa literatura encontrada para el rango nanométrico, se desprende un primer análisis sobre las características de deformación a altas temperaturas desarrollado por Gutiérrez Mora y col. (2) y por Lorenzo Martín y col. (3). Aunque se apunta que el mecanismo de deformación sea el deslizamiento de fronteras de grano, no se concluye cuál es el mecanismo de acomodación. Igualmente interesante resulta estudiar la influencia de la fase vítrea en los mecanismos de deformación plástica, aspecto éste que no ha sido estudiado.

\section{PROCEDIMIENTO EXPERIMENTAL}

En las muestras de cerámicos nanométricos de YTZP con diferentes cantidades de fase vítrea: $5 \%$ y $10 \%$ en peso, se realizó un análisis químico. La fase vítrea contiene un $80 \%$ en peso de $\mathrm{SiO}_{2^{\prime}} 15 \%$ en peso de $\mathrm{Al}_{2} \mathrm{O}_{3^{\prime}}$ y $5 \%$ en peso de SrO. Las densidades experimentales están todas por encima del $97 \%$ del valor teórico $\left(6.1 \mathrm{gcm}^{-3}\right)$. Denominaremos muestras "puras" aquellas que no contienen fase vítrea.

Se realizaron ensayos de deformación a carga constante (fluencia), con tensiones comprendidas entre 5 y $35 \mathrm{MPa}$, en un rango de temperaturas de $1150-1200^{\circ} \mathrm{C}$, con las consiguientes velocidades de deformación $\left(10^{-7} \mathrm{~s}^{-1}-10^{-5} \mathrm{~s}^{-1}\right)$. El rango de temperatura escogido, es suficiente- mente alto para garantizar que la difusión juegue un papel importante en el mecanismo de deformación, pero a su vez suficientemente bajo para evitar el crecimiento dinámico de los granos.

Con el objeto de realizar un análisis microestructural del material se prepararon muestras para ser estudiadas por Microscopía Electrónica de Transmisión (TEM), para lo cual se cortaron láminas de las muestras antes y después de ser deformadas. Dichas láminas fueron adelgazadas hasta la transparencia electrónica siguiendo el procedimiento convencional descrito en (3); y observadas en el microscopio de transmisión (Philips CM200) operando a 200kV. Se tomaron las micrografías necesarias para hacer un estudio preliminar del tamaño de grano de cada muestra con el objeto de ver si éste cambia cuando las muestras son deformadas por fluencia.

\section{RESULTADOS}

Los datos que se desprenden de los ensayos de fluencia se ajustan a la ecuación constitutiva de fluencia a alta temperatura:

$$
\dot{\varepsilon}=\mathrm{A} \frac{\sigma^{\mathrm{n}}}{\mathrm{d}^{\mathrm{p}}} \exp \left(\frac{-\mathrm{Q}}{\mathrm{kT}}\right)
$$

donde A, es una constante; $\sigma$, es la tensión; $\dot{\varepsilon}$ la velocidad de deformación; $\mathrm{k}$, la constante de Boltzmann; T, la temperatura absoluta; n, el exponente de tensión y $\mathrm{Q}$, la energía de activación. Estos dos últimos parámetros $(\mathrm{n}, \mathrm{Q})$ son característicos del mecanismo de deformación. El cálculo de $\mathrm{n}$ se hace mediante saltos de tensión y de velocidad de deformación para las mismas condiciones de deformación a temperatura constante. El cálculo de $Q$ es análogo pero se mantiene la tensión constante para saltos de temperatura (3). 
Con el conjunto de valores obtenidos para n y $\mathrm{Q}$, se calculan los valores medios de dichos parámetros para cada muestra. Dichos valores de $\mathrm{n}$ y $\mathrm{Q}$ en función de la concentración de fase vítrea se recogen en la Tabla I.

TABLA I: TABLA DE $\mathrm{n}$ Y Q EN FUNCIÓN DE LA CONCENTRACIÓN DE FASE VÍTREA.

\begin{tabular}{|c|c|c|}
\hline muestra & $\mathbf{n}$ & $\mathbf{Q}(\mathbf{k J} / \mathbf{m o l})$ \\
\hline Pura & $2.0 \pm 0.4$ & $682 \pm 15$ \\
\hline $5 \%$ fase vítrea & $2.2 \pm 0.5$ & $695 \pm 29$ \\
\hline $10 \%$ fase vítrea & $2.3 \pm 0.5$ & $711 \pm 29$ \\
\hline
\end{tabular}

A partir de estos valores y con el análisis microestructural de las muestras por TEM, se puede determinar el mecanismo de deformación predominante. Al observar las muestras de YTZP sin fase vítrea por TEM antes y después de ser deformadas, no se aprecian cambios aparentes en la microestructura de las mismas, tal como se deduce de la figura 2. El tamaño medio de grano se mantiene constante y no aparecen grietas ni cavitación alguna. A partir de un conjunto de micrografías se ha estimado el tamaño medio de grano de 80-100 nm en todas las muestras.

\section{DISCUSIÓN}

De los análisis y ensayos realizados se deduce que el mecanismo de deformación es de deslizamiento de fronteras de grano. El valor del exponente de tensión es el mismo medido en este material en el caso de tamaños de grano de escala submicrométrica (1).

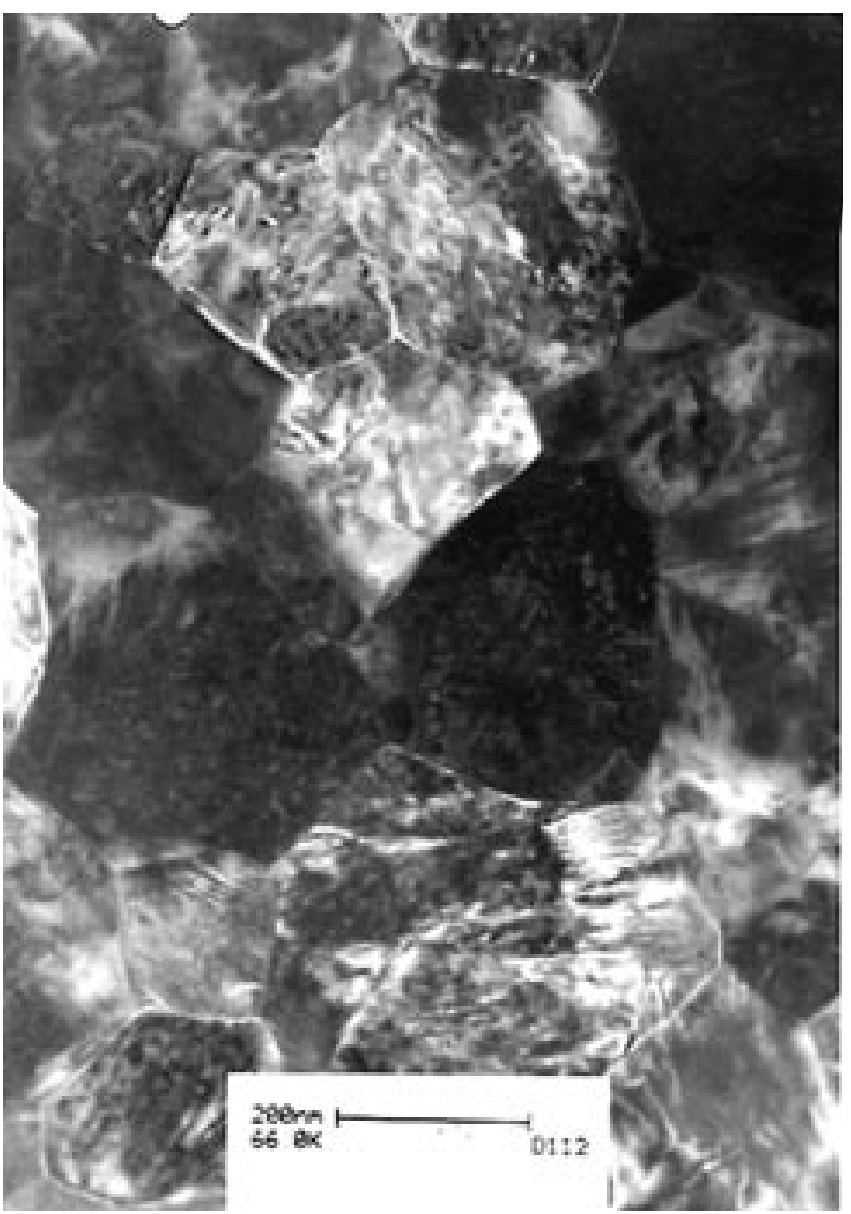

Figura 2B: muestra pura después de ser deformada

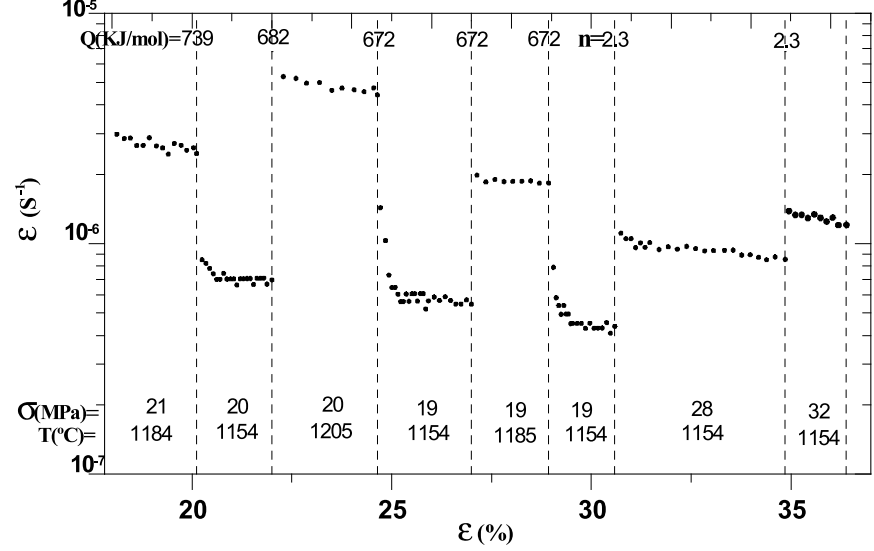

Figura 1: Curva de fluencia para la muestra con un $5 \%$ de fase vítrea. Ejemplo del cálculo del exponente de tensión y la energía de activación

Por el contrario, resulta particularmente relevante que no exista tensión umbral en estos materiales nanométricos. Este hecho está conforme con los resultados de la literatura publicados en muestras submicrométricas con presencia de fase vítrea (1). Sin embargo, en el caso de las muestras puras, la existencia de una tensión umbral en muestras submicrométricas está bien documentada. La razón por la cual no existe tensión umbral en ningún caso en las muestras nanométricas es objeto de intenso estudio (4).

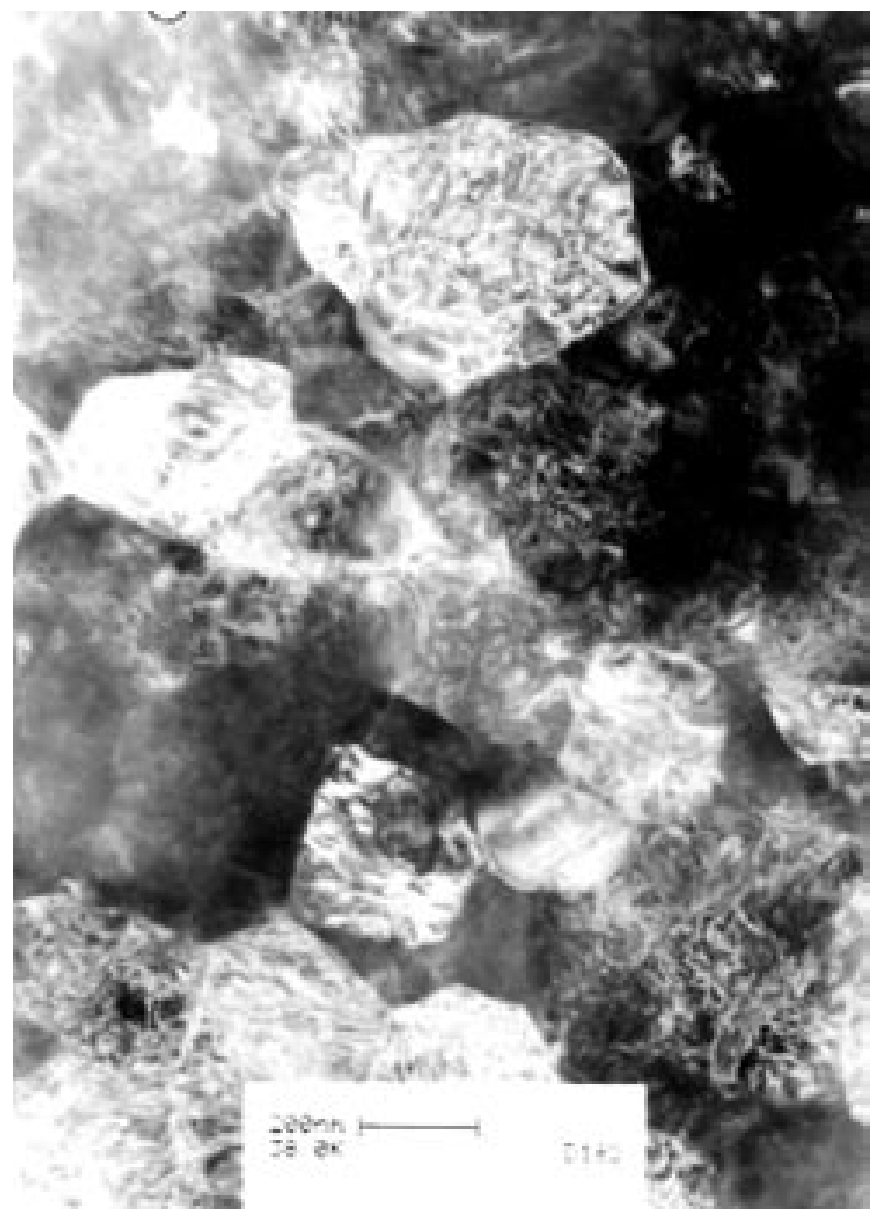

Figura 2A: Muestra pura antes de ser deformada 
Con respecto a los resultados de la energía de activación, ésta resulta ser significativamente más alta que el valor aceptado para la energía de activación del coeficiente de difusión catiónico. Este resultado está de acuerdo con predicciones recientes existentes en la literatura (5) que apuntan a un significativo aumento del valor medido de la energía de activación aparente de los policristales de YTZP a escala nanométrica.

Finalmente, el papel de la fase vítrea es esencial en lo que concierne a la resistencia mecánica de estos materiales. Lorenzo-Martín y col. han publicado una dependencia notable de dicha resistencia con la fase vítrea medida a través de ensayos de deformación a velocidad constante (3). Dicha disminución de la resistencia está en buen acuerdo con los resultados publicados por (6) en este mismo material a escala submicrométrica con fases vítreas de naturaleza química diferente. Hay estudios en desarrollo para intentar explicar de forma definitiva las diferencias encontradas entre el comportamiento mecánico a la escala nanométrica y el correspondiente a la escala submicrométrica.

\section{CONCLUSIONES}

El mecanismo de deformación de los materiales cerámicos nanométricos de YTZP es el deslizamiento de fronteras de granos, ya que este mecanismo es consistente con las observaciones microestructurales de las muestras deformadas. Las diferencias observadas entre el comportamiento a escala nanométrica y el descrito a escala submicrométrica está siendo objeto de estudio.

\section{AGRADECIMIENTOS}

Los autores agradecen la ayuda financiera aportada por la "Comisión Interministerial de Ciencia y Tecnología" a través del proyecto MAT2000-0622.

\section{BIBLIOGRAFÍA}

1. M. Jiménez-Melendo, A. Domínguez-Rodríguez, "High temperature characteristic of superplastic yttria-stabilized zirconia. An examination of the flow process". Acta mater, 2000,48,3201.

2. F. Gutiérrez-Mora, A. Domínguez-Rodríguez, M. Jiménez-Melendo, “Creep of nanocrystalline YSZP ceramics" ,Nanostruct. Mater; 1999,11,pp.531-37.

3. C. Lorenzo-Martín, J. A. Flores-Vázquez, D. Gómez-García, A. Muñoz-Bernabé, Danz-Zhiou, J. Gómez-Herrero y A. Domínguez-Rodríguez, “Mechanical behaviour of YTZP nanocrystalline ceramics as a function of the glassyphase content". Journal of the European Ceramic Society (vol 22, 14-15, 2002, pp. 2603-2607).

4 D. Gómez-García, C. Lorenzo-Martín, A. Muñoz-Bernabé and A. Domínguez-Rodríguez; "Correlation between yttrium segregation at the grain boundaries and the threshold stress for plasticity in yttria tetragonal zirconia polycrystals", Philosophical Magazine A, 2003, Vol.83, No.1,93-108

5. D. Gómez-García, C. Lorenzo-Martín, A. Muñoz-Bernabé and A. Domínguez-Rodríguez; " Model of the high-temperature plastic deformation of nanocrystalline materials: Application to yttria tetragonal zirconia", Physical review B 67, 144101 (2003).

6-M. Gust, G. Goo, J. Wolfenstine and M. Mecartney; Influence of amorphous grain boundary phases on the superplastic behaviour of $3 \mathrm{~mol} \%$-yttriastabilized tetragonal polycrystals (3Y-TZP). J. Am. Ceram. Soc; 1993, 76(7), 1681-1690.

Recibido: 1.2 .03

Aceptado: 30.11 .03 